\title{
FUZZY LOGIC BASED IMPROVED MEDIUM ACCESS CONTROLLER FOR CONGESTION CONTROL AND PREDICTION THE SYSTEMS IN WSNS
}

\author{
M. Sri Lakshmi ${ }^{* 1}$, Dr. J. Keziya Rani ${ }^{* 2}$ \\ ${ }^{1}$ Research Scholar, Department of Computer Science \& Technology, S.K University, Anantapuramu, Andhra Pradesh, India. \\ ${ }^{2}$ Assistant Professor, Department of Computer Science \& Technology, S.K University, Anantapuramu, Andhra Pradesh, India. \\ EmailID:I'mari_srilakshmi67@yahoo.co.in, ${ }^{2}$ kejiyaraj@gmail.com
}

\begin{abstract}
: in resource-constrained networks, particularly those with limited bandwidth to manage high-volume data transmission, network congestion is a major issue, resulting in poor quality of service, including packet loss and delay throughput. Due to self-contained batteries that limit sensor node lifetime, this issue is important in wireless sensor networks (WSNs) with limitations and restrictions, such as limited processing power, memory, and transmission. By determining a path that avoids congested highways, the network can be extended. As a result, we present a WSN route determination architecture that is congestion-aware. The architecture is divided into three stages: In a top-down hierarchical structure, the first path is created. Energy-aware assisted routing for route derivation. Exponential smoothing is used to forecast congestion, but the final parameters for route determination are not taken into account. We use fuzzy logic systems to evaluate proper weights for a variety of factors, including shop count, remaining energy, buffer occupancy, and forwarding rate, as well as a bat algorithm to optimize the weight over the membership functions. Eventually proposed model shows the high throughput, low packet loss, save energy, and extending network lifetime.
\end{abstract}

Keywords: WMSN, Sensor nodes, fuzzy logic system, MAC, Membership function.

\section{Introduction}

There are the challenges of maintaining message latency and data integrity and maintaining the battery life of sensor nodes within wireless sensor networks (WSNs). This research aims to propose a fuzzy logic algorithm to tackle this issue, which is hard to cope with using traditional techniques. This research aims to employ a fuzzy logic programme to deal with energy consumption and minimise packet loss. This paper demonstrates the exactly how fuzzy logic might be used to resolve the problem efficiently with a unpretentious method.The most urgent customers are given priority over those who arrive later; regardless of how long they have been waiting. Which are contradicting; they must maintain and maintain at the same time. In this context, our study's major goal was to propose a control algorithm for priority and transmission rate using an intelligent adaptive controller. This study provides the performance indicators concerning fuzzy variables It aimed the queue length and traffic rate at giving more importance to nodes that drop more packets and use more energy. This abstract is as follows. Section II introduces the related research. Section III derives the system model and demonstrates the mathematical approach. Section IV summaries the proposed algorithm and its performs evaluations. This section concludes this paper.

\section{Related work}

Based on our research's fuzzy logic method, a range of algorithms have been developed to ensure optimal network performance. Ramesh et al. [1] used an unknown set method to quantify the expected cost of a fuzzy batch arrival queuing model.

In the QoS service model, Ashok Kumar [2] developed a nonlinear programming method for deriving the membership functions of steady-state performance measures. The definition is inspired by Zadeh's[3] extension theory. To test machine efficiency, two sets of quantitative nonlinear programmes are used. In energy-harvesting WSNs, Chen et al. [4] suggested a dynamic adaptive MAC system. They've come up with a useful bound for the back-off window. The slotted CSMA/CA parameters can be dynamically modified using a fuzzy algorithm, and the parameters are also influenced by the network's energy-harvesting mode.

Many authors suggest that Fuzzy Queuing could measure critical incidents. Authors from [7-9] developed the membership function of the performances in their algorithms. They created an algorithm that maximises service quality while keeping the time, energy, and packet drop constraints.

Kaur and Kumar et al. [10] use the nodes' flexibility and heterogeneity as restrictions in their primary-based, application-specific congestion control clustering protocol 
(PASCC). The type and priority of the data collected by PASCC are used to assess congestion in WSNs. The distance determines the importance of packets that the device must travel. $\mathrm{CHs}$ also ensure that the services received from higher layers support the lower layers to the maximum extent possible. During congestion, it prioritises packets based on their value and arrival time. PASCC increases network efficiency by extending network lifespan, reducing energy usage, and enhancing data transmission quality.

The author of [11] Recommended that transit data be replaced with data collected locally. They came up with congestionaware routing, which searches out more efficient routes. On the other hand, a fuzzy rate controller decides on the best threshold based on two parameters: buffer occupancy and congestion ranking. This paper established the Exponential Weight of Priority Dependent Rate Control (EWP-PBC) algorithm (ARC) for adaptive rate control. This is discriminatory means since different data have different definitions. Fusion has identified three interventions to address urban traffic congestion. In the first mechanism, a child transmits after its parent stops transmission. Muthuselvi Mayandi and Kavitha Velayudhan Pillai [15] suggested EWPBR prioritises traffic light assignments using the input and output data rates. Because it is not possible to detect congestion from the usage of the buffer overflow occurs, which indications to packet loss, reduce in packet size and the very delayed data delivery. The likelihood technique regulates all nodes' rates at the same rate. WMSN nodes can be deployed at different locations depending on their value, necessitating differential operation.

\section{Materials and methods}

\section{Fuzzy Based Enhanced Medium Access Control Protocol}

WSN's basic structure is depicted in Figure 1. One base station and nine sensor nodes (S1-S9) make up this network (BS). Each sensor node gathers data from its environment and transmits it to the BS. By managing all sensors, BS acts as the network's coordinator. The MAC protocol controls information packet traffic congestion in the network. The project's body sensors are expected to have a short lifespan. The collected data packet will be forwarded to the BOSS department by the end of this month. It would be harmed otherwise. The MAC protocol for WSN is created on the super-frame that is illustrated in Figure 2.

\subsubsection{Network Topology Creation}

As sensor nodes receive data and transmit it to the BS, they gather information from the surroundings (BS). Passing the data via the communication channel may clash with the data packets. Colliding packets are then retransmitted, and this will lead the network to high electricity depletion. Therefore, congestion of WSN data packets will be managed by finetuning the MAC protocol. By integrating fuzzy logic, also enhances the MAC protocol. Fuzzy threshold method prioritization process for the delay, buffer occupancy, available buffer room. The value depends on Incoming Packet Data Rate, Residual Delay and buffers occupancy. Figure 3 depicts the workflow of the approach of this study.

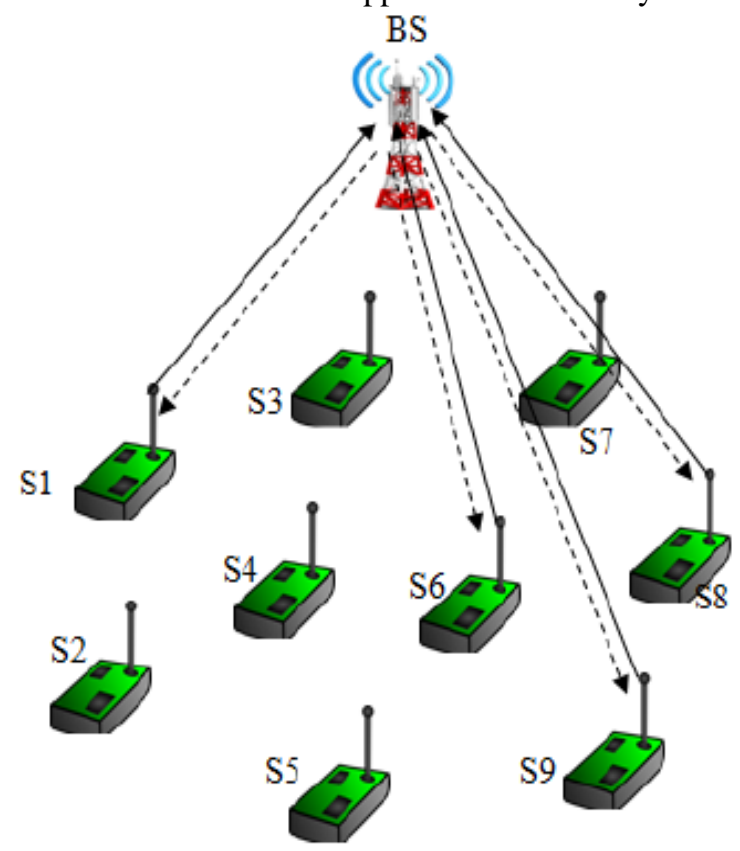

Figure 1. Network Model

3.1.2 Flow model:

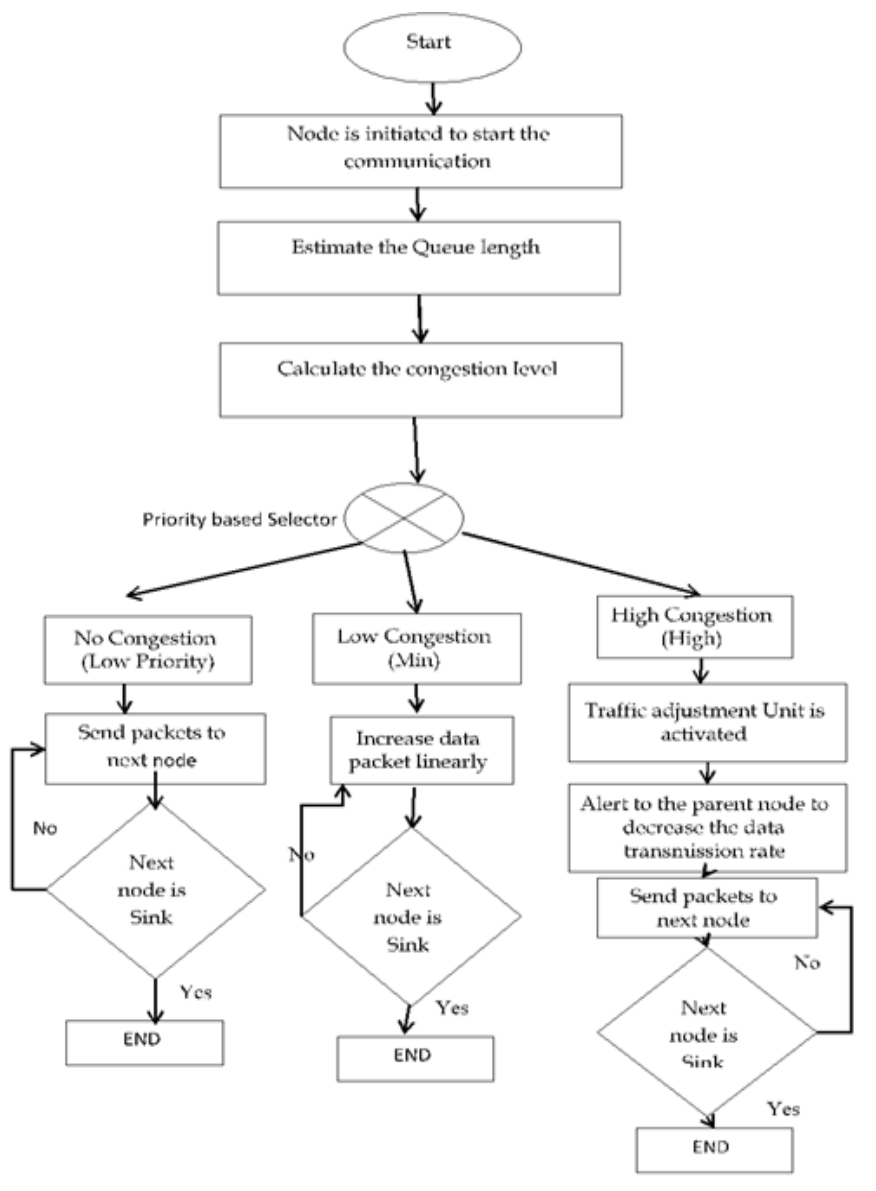

Figure 2. Proposed Work Flow model 
Our proposed model is based on three congestion detection indictors, such as: 1) Buffer occupancy, 2). Packet deliver ratio of the sensor node, 3) Delay of the sensor node

Buffer occupancy: it is an important metrics at the time of routing a packet from one hop to another hop. If the parent node has higher buffer occupancy, then congestion probability will also be high.

Packet deliver ratio of the sensor node: it is the essential metrics for detecting the congestion. It is the ratio of incoming to the outgoing packets within the time window. The proposed system uses sliding window for transmitting a packet. When number of packets transferred exceeds window size then packet loss will occur and it decreases throughput of a network.

Residual Delay: it is the key metrics for detecting the congestion. It shows an amount of time taken by the SN to transmit the packets from one hop to another hop.

Figure 3. Illustrations the construction of fuzzy system

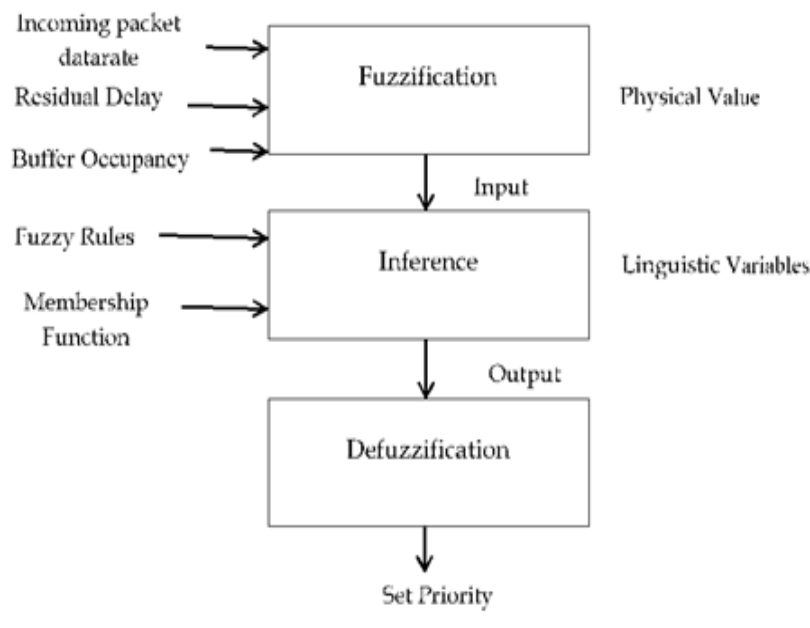

Figure 3. The fuzzy logic system.

In this model, the incoming data rate, residual delay, and BO are given as input to the model. Residual uncertainty is the difference between the packet's cumulative delay and the router's extreme delay tolerance. For this example, data packets will be collected starting at time $\mathrm{t}=0$.

$$
R D_{P k t s}(t)=d_{\text {Max }}-d_{\text {cum }}^{i}(t)
$$

Where maximum delay on packets from $i^{\text {th }} \mathrm{SN}$ to the BS. The proportion between the collected packs and the buffer size is calculated by multiplying the buffer size by the buffer occupancy percentage.

$$
B O_{P k t s}(t)=\frac{S_{P k t s}^{i}(t)}{S_{\text {buff }}^{i}}
$$

Where $\mathrm{S}$ is the number of $\mathrm{SN}$, and $\mathrm{T}$ is the overall count of the nodes. These crisp values or input variables are input into a fuzzy logic system, and the system converts the values into fuzzy variables.
The proposed Improved MAC Algorithm links traffic rate measurement and dynamic queue management to attain energy competence and expand QoS. We intend to project a fuzzybased MAC algorithm that has better performance for minimising packet loss. The dynamic schedule guarantees this to maintain the conversation. We will also be diagnosing the traffic flow at the queue level. To characterise all linguistic factors, membership functions are needed. A triangular form will be related with the inputs, and a singleton function will be involved for the outputs shown in figure 4.

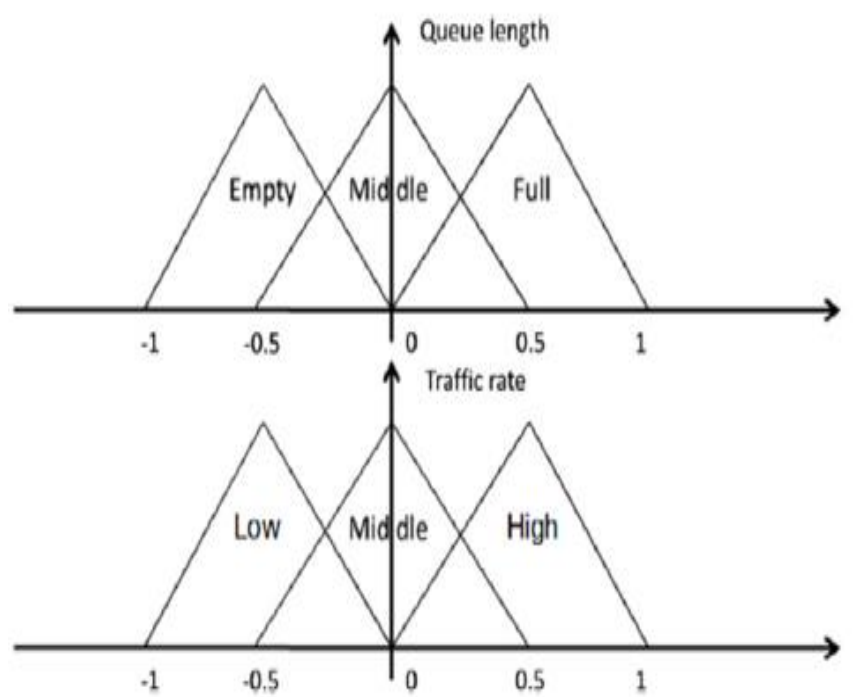

Figure 4. Membership functions for the linguistic parameters

Three levels result from changing priority levels: low priority (1), middle priority (2), and high priority (3). (3). The point illustrated in figure 3 is that there will not be any significant output linguistic variables variation.

First, we ought to map two sets in the fuzzy rule.

Queue-Length $=\{$ Empty, Medium, Full $\}$

Traffic-Rate $=\{$ Low, Medium, High $\}$.

Fuzzification: In this obtaining the crisp inputs from the preferred input variables and estimating the degree to which the inputs fit in to each of the apt fuzzy set. The original estimations of and were converted into a fuzzy dynamic system. Then for each fuzzy variable, a participant work is determined. In this model, the packet prioritisation parameter is used. Fuzzy variables are low (L), medium $(\mathrm{M})$ and high $(\mathrm{H})$ in the range from 0 to 100 secs. Labels for fuzzy variables include $\mathrm{L}, \mathrm{M}$ and $\mathrm{H}$ in the prolong.

[0, 1 and 2 respectively]. The FIS uses three linguistic variables High $(\mathrm{H})$, Medium $(\mathrm{M})$ and Low $(\mathrm{L})$ for detecting the Congestion Level (CL). Where ' $\mathrm{CL}$ ' is calculated as summing up all the weight of fuzzy classes L,M and H. Fuzzy factors of the yield Packet Priority (PP) are High (H), Average (AV) and Low (L) as seemed in Table 1. The defuzzification is the last step in FIS, this model used centroid defuzzifier for 
transforming inferred output to a crisp output value. It is represented in Equation (5)

$$
\mathrm{O}=\sum_{r=1}^{18} C L
$$

After applying the centroid defuzzifier, the crisp value of $\mathrm{O}$ lies between 0 to 6 .

If-then rule will be used to work out the fuzzy factors to achieve the fuzzy sets. The standards are more reliable due to diverse information sources (AND). The administrator tends to allocate scholarships that have the least amount of applicants, keeping in mind administrative difficulties. For a practical example, if the following criteria met (low or high), the consequence was 0 .

Defuzzification: In the defuzzification step, the centre of gravity method should be applied. It bases this idea on the estimation of maximum values for subunits. The argument value of the variable (Zmoy) is calculated by using equation (6). The $\mathrm{Pk}$ arguments reflect the calculations made at the inference stage, as shown in Figure 3.

$\mathrm{Zmoy}=\mathrm{Z} 1 \mathrm{P} 1+\mathrm{Z} 2 \mathrm{P} 2 \mathrm{P} 1+\mathrm{P} 2$.

The input weight values correspond to the trial inputs. The value " $Z$ " defines the action of enforcing the rule. This variable falls within the interval $[-1,1]$.

Possibility of the total states is $33=$. (27) In each of these examples, the choice of the fuzzy output variable will be significant.

Table 1. Priority based Fuzzy indexed

\begin{tabular}{|l|l|l|l|l|}
\hline Index & $B O_{P k t s}$ & $D R_{P k t s}$ & $R D_{P k t s}$ & CL \\
\hline 1 & L & L & L & 0 \\
\hline 2 & L & L & M & 1 \\
\hline 3 & L & L & H & 2 \\
\hline 4 & L & M & L & 1 \\
\hline 5 & L & M & M & 2 \\
\hline 6 & L & M & H & 3 \\
\hline 7 & L & H & L & 2 \\
\hline 8 & L & H & M & 3 \\
\hline 9 & L & H & H & 4 \\
\hline 10 & M & L & L & 1 \\
\hline 11 & M & L & M & 2 \\
\hline 12 & M & L & H & 3 \\
\hline 13 & M & M & L & 2 \\
\hline 14 & M & M & M & 3 \\
\hline 15 & M & M & H & 4 \\
\hline 16 & M & H & L & 3 \\
\hline 17 & M & H & M & 4 \\
\hline 18 & M & H & H & 5 \\
\hline 19 & H & L & L & 2 \\
\hline 20 & H & L & M & 3 \\
\hline 21 & H & L & H & 4 \\
\hline 22 & H & M & L & 3 \\
\hline
\end{tabular}

\begin{tabular}{|l|l|l|l|l|}
\hline 23 & H & M & M & 4 \\
\hline 24 & H & M & H & 5 \\
\hline 25 & H & H & L & 4 \\
\hline 26 & H & H & M & 5 \\
\hline 27 & H & H & H & 6 \\
\hline
\end{tabular}

The triangular functions are used in most of the applications to manage computational complexity in an efficient manner. The triangular functions are used to represent the membership function in a simple way. Fuzzy values are evaluated through membership function of the fuzzifier for example if the buffer occupancy of the parent node is 25 , then the corresponding is $[0,1,0]$ as can be seen in Figure 5. For example if the packet delivery ration of the node is 4 , then the corresponding is [0, $0.5,0.5$ ] as can be seen in Figure 6. For example if the delay of the node is 1.10 , then the corresponding is $[0,0.5,0.5]$ as can be seen in Figure 7. The fuzzy implication step combines the fuzzy rules from the fuzzy rule base. The fuzzy rule base is constructed to control the output variables. The fuzzy IFTHEN rule is simple and contains a condition and a conclusion. The proposed model takes 3 fuzzy variables and 3 classes hence the fuzzy rule base has $=27$ rules. The output of the each rule is mapped to a corresponding consequence class ' $C$ '. The syntax of the rule is

$r=$ if $B O_{r}$ is $\mathrm{BO}^{\mathrm{FZ}}$ and $D R_{r}$ is $\mathrm{DR}^{\mathrm{FZ}}$ and $R D_{r}$ is $\mathrm{RD}^{\mathrm{FZ}}$ then output is $\mathrm{C}$

Then output is $\mathrm{C}$

Where FZ is one of the fuzzy classes L or H or M. Table 5.1 is used to describe these 18 rules as various level of congestion in the WMSNs. The membership function of Consequence Class is shown in the Figure 8.

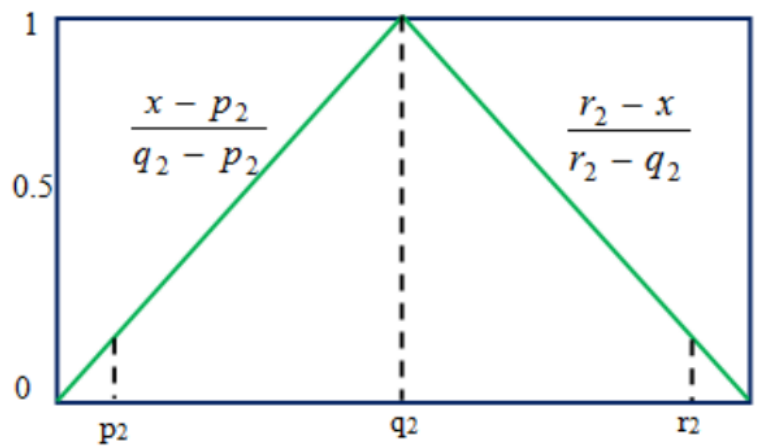

Figure 5. Basic structure of triangular function

For improved learning outcomes, the current school size is utilized. The limit and middle of the road capacities are used in the triangular function for the organization's dependable advancement. The triangle participation work for information esteem y is evaluated, and triangles participation work applied to all phonetic qualities or fuzzy statistics of a standard utilizing the capacities. (8).

$$
f\left(y ; p_{2}, q_{2}, r_{2}\right)=\max \left[\min \left[\frac{y-p_{2}}{q_{2}-p_{2}}, \frac{r_{2}-y}{r_{2}-q_{1}}\right], 0\right] \text { (8) }
$$




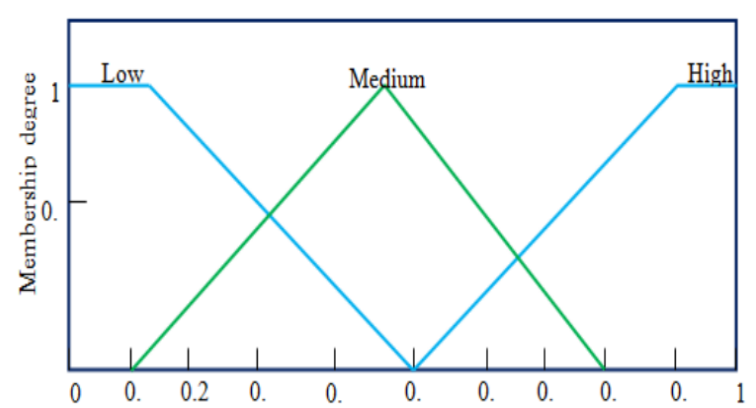

Figure 6. Buffer Occupancy

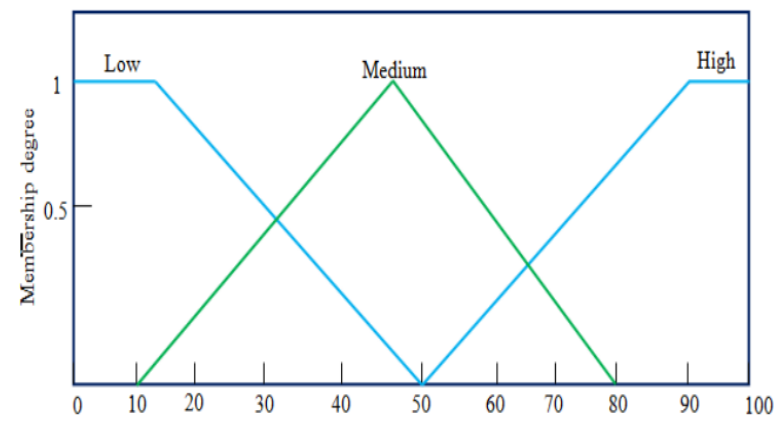

Figure 7. Membership function of Residual delay

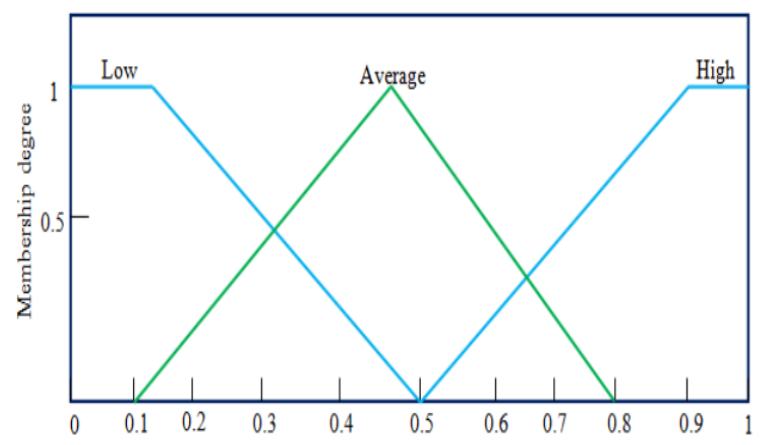

Figure 8 Packet Priority

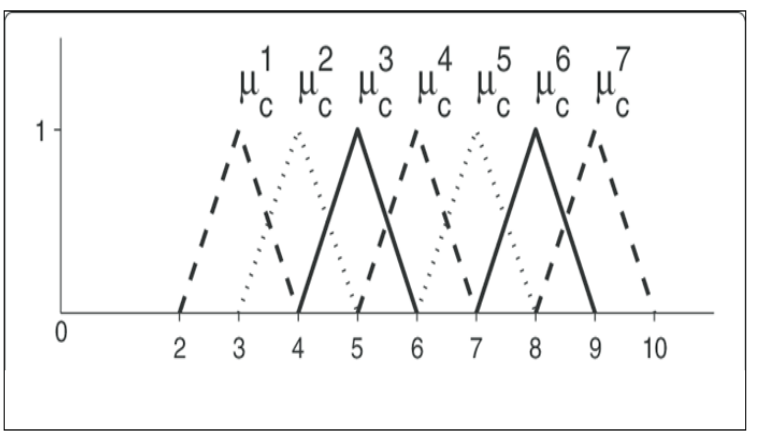

Figure 9. Membership function of Consequence Class

\section{FLIMAC Classification}

FLIMAC classifies the Level of Congestion (CL) into three classes: Not Congested (NC), Lightly Congested (LC) and Heavily Congested (HC) by using minimum [CTH(min)] and maximum $[\mathrm{CTH}(\max )]$ threshold values. There is no need to apply a congestion control algorithm for NC classes because it has sufficient resources to handle the network traffic. FLIMAC congestion control algorithm is started at LC or HC level networks.

\subsection{Algorithm 1- FLIMAC Congestion prediction Algorithm}

1. Let $\mathrm{BO}_{\mathrm{pkts}}$ be packets buffer occupancy of the sensor node

2. Initialize $\mathrm{C}_{\mathrm{TH}}(\mathrm{min})$ (Minimum Congestion threshold and $\mathrm{C}_{\mathrm{TH}}(\max )$ ( maximum Congestion threshold)

3. Ready to send packets

4. Calculate congestion level using FIS

5. If $\left(\mathrm{BO}_{\mathrm{pkts}}<\mathrm{C}_{\mathrm{TH}}(\min )\right)$

6. $\mathrm{CL}($ Congestion Level $)=0$

7. Send packets to next node

8. If (next node $==\operatorname{sink}$ )

9. Insert incoming packets $\left(\mathrm{D}_{\mathrm{i}}\right)$

10. End if

11. Else repeat steps 7 to 10

12. Else if $\left(\mathrm{C}_{\mathrm{TH}}(\min )<=\mathrm{BO}_{\mathrm{pkts}}<\mathrm{C}_{\mathrm{TH}}(\max )\right)$

13. $\mathrm{CL}$ (congestion level) $=\mathrm{Min}$

14. Increase data transmission rate linearly

15. repeat steps 7 to 10

16. Else $\left(\mathrm{BO}_{\mathrm{pkts}}>\mathrm{C}_{\mathrm{TH}}(\max )\right)$

17. $\mathrm{CL}$ (congestion level) $=\mathrm{Max}$

18. TAU (Traffic Adjustment Unit )is activated

19. Inform congestion level to its parent nodes

20. Parent nodes decrease data transmission rate

21. Repeat steps 19 to 20 until reach source

22. Repeat steps 7 to 11

23. End if

In the proposed protocol the traffic rate of packets are adjusted at Traffic Adjustment Unit (TAU) based on the level of congestion. If the packets buffer occupancy of the sensor node is lower than the lowest threshold then probability of congestion is zero. On the other end the packets buffer occupancy of the $\mathrm{SN}$ is higher than the extreme threshold then TAU is activated and notifies the congestion to its upstream nodes. After receiving notification from TAU, the parent nodes reduce their transmission rate. If buffer occupancy of the sensor node is above minimum threshold and below maximum threshold then probability of congestion is low and $\mathrm{SN}$ increases its transmission rate linearly. The congestion prediction algorithm is defined in Algorithm 1.

\subsection{Network Model}

Figure 10 shows a portion of the network represented in our model. The dashed circle represents the communication range of this node with neighbouring nodes $(\mathrm{NN})$. The point be contingent on the format of the message. The network will be generally built into layers based on the hops count. Once the path is fixed, the source node will hand it over therefore 
(commencing the 20th SN, examine 18, 9, and 4, and to end go to the sink node)

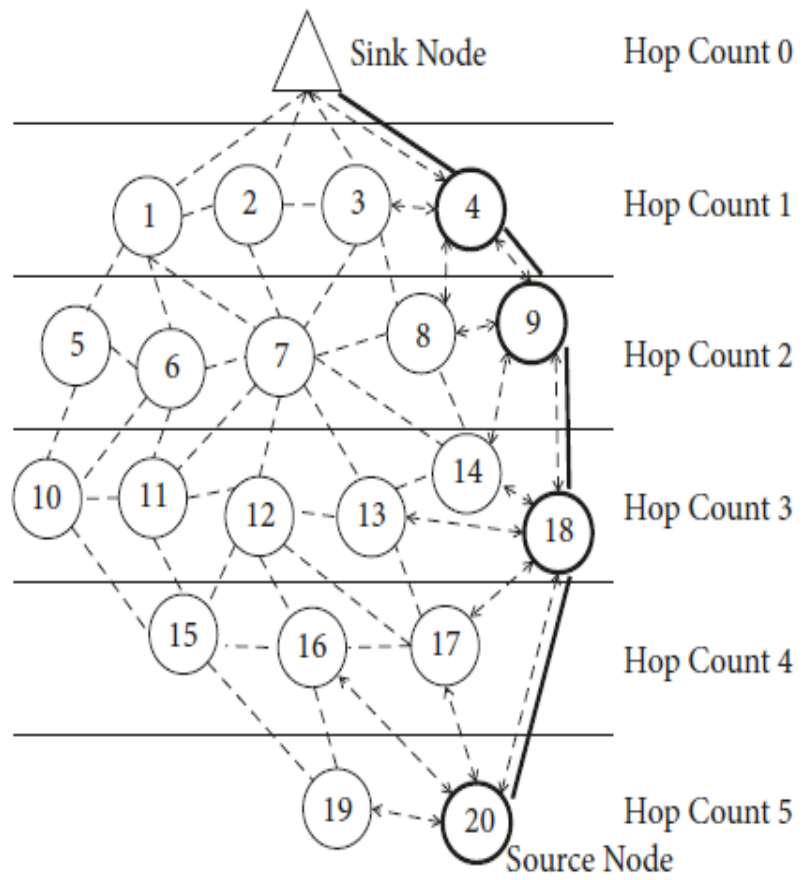

Figure 10 Example: network model

\subsection{Congestion Estimation and Data Rate Adjustment}

At the time " $t$ " the congestion level of sink node can be measured by using congestion prediction algorithm 1 .

The new retiring rate of each child node "a" of sink () can be measured mathematically by using the equation (9) and forwarded to the set of child nodes of the sink.

$$
O D^{a}{ }_{\text {new }}=O D^{a}{ }_{\text {new }}-O D^{a}{ }_{\text {new }}\left(\mathrm{CL}_{\text {sink }} *\left(1-\frac{\mathrm{TP}^{a}}{T P_{\text {sink }}}\right)\right.
$$

At the time " $t$ " the congestion level of parent node can be measured by using congestion prediction algorithm 5.1. The new outgoing rate of each child node "b" of parent ( ) can be measured mathematically by using the Equation (10).

$$
O D_{\text {new }}^{b}=O D_{\text {new }}^{b}-O D_{\text {new }}^{b}\left(\mathrm{CL}_{\text {sink }} *\left(1-\frac{\mathrm{TP}^{b}}{T P^{a}}\right)\right.
$$

The outgoing rate of each child node may be determined by its parent nodes. The sink node has no parent node and hence it is determined by taking inverse of average service time.

\section{Results and Discussion}

To detect the level of congestion of each sensor node, the existing system such as EWPBRC and FEWPBRC comprises nodes incoming and outgoing data rate. The FLIMAC includes incoming, outgoing and buffer occupancy of the node. The existing work did not include other QoS parameters like delay and packet delivery ratio. Our proposed work predicts the congestion by using fuzzy inference system constructed on incoming and outgoing data rate along with buffer occupancy of the parent node, packet delivery ratio of the sensor node and

delay of the sensor node. The minimum and maximum threshold value is used to detect the buffer occupancy at run time. Based on the congestion intensity the outgoing data rate of the node is minimized which decreases number packet drops. The basic network model is described in Figure 10, in which the resources are evenly distributed to the $\mathrm{SN}$ based on priority of source and geographical location which gives effective throughput. The present work Simulated by using NS2 simulator.

Table 2. The simulation parameters

\begin{tabular}{|l|l|}
\hline Area of WSN & 500 X 500m \\
\hline Number of nodes & 100 \\
\hline Number of sink & 01 \\
\hline Packet size & 200 bytes \\
\hline Routing protocol & AODV \\
\hline Sensor node- buffer size & 50 packets \\
\hline Sink node-buffer size & 100 packets \\
\hline Simulation time/runs & 60 secs/20 runs \\
\hline
\end{tabular}

The simulation parameters are presented in table 2. for the experiment simulation area consider as 500X500 m, Number of Sensor nodes consider are 12 among this 11 Nodes are Sensor nodes and 1 node is acting as Sink node or a Base station in the network transmission range is 50 meters, packet size of the sensor node is 50 packets and sink node capacity to receive the packets is 100 packets. Each node's initial energy is assigned 10 Joules, for routing it uses AODV protocol. Inference queue is Drop tail and simulation time per number of runs 75 Seconds/20 Runs.

Table 3 Comparative analysis on delay

\begin{tabular}{|l|l|l|l|}
\hline No.of Nodes & FLIMAC & EWPBRC & FEWPBR \\
\hline 10 & 0.32 & 0.33 & 0.34 \\
\hline 20 & 0.29 & 0.36 & 0.35 \\
\hline 30 & 0.35 & 0.35 & 0.36 \\
\hline 40 & 0.31 & 0.33 & 0.35 \\
\hline 50 & 0.33 & 0.34 & 0.35 \\
\hline 60 & 0.28 & 0.36 & 0.35 \\
\hline 70 & 0.31 & 0.32 & 0.34 \\
\hline 80 & 0.27 & 0.34 & 0.34 \\
\hline 90 & 0.26 & 0.31 & 0.34 \\
\hline 100 & 0.33 & 0.34 & \\
\hline
\end{tabular}


Graph 1. Comparative analysis on delay

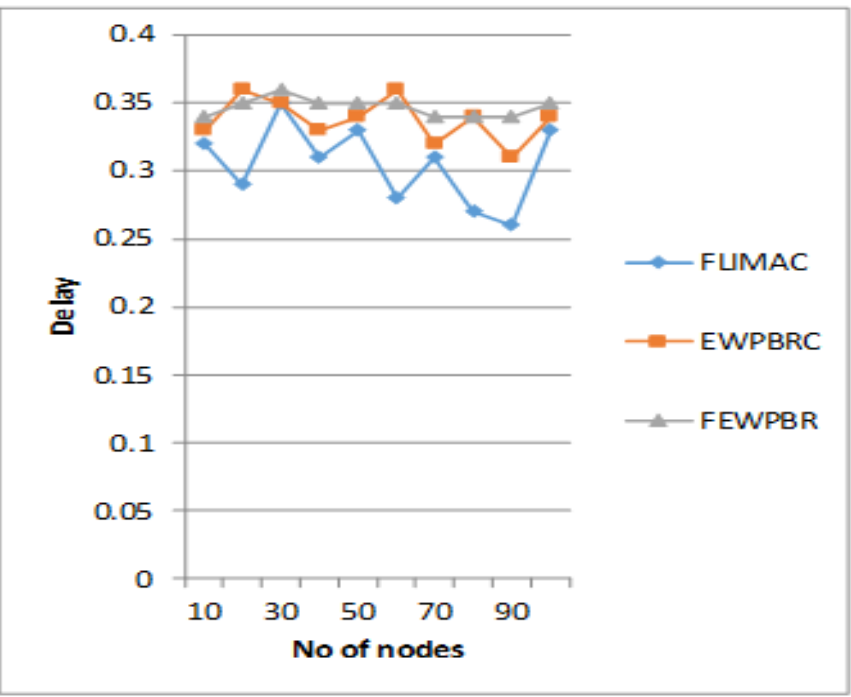

Table 4: Comparative analysis on Throughput

\begin{tabular}{|l|l|l|l|}
\hline $\begin{array}{l}\text { No.of } \\
\text { Nodes }\end{array}$ & FLIMAC & EWPBRC & FEWPBR \\
\hline 10 & 320 & 270 & 290 \\
\hline 20 & 345 & 318 & 340 \\
\hline 30 & 380 & 325 & 340 \\
\hline 40 & 320 & 290 & 290 \\
\hline 50 & 375 & 280 & 310 \\
\hline 60 & 345 & 295 & 340 \\
\hline 70 & 375 & 280 & 310 \\
\hline 80 & 300 & 290 & 300 \\
\hline 90 & 340 & 330 & 335 \\
\hline 100 & 378 & 320 & 330 \\
\hline
\end{tabular}

Graph 2. Comparative analysis on Throughput

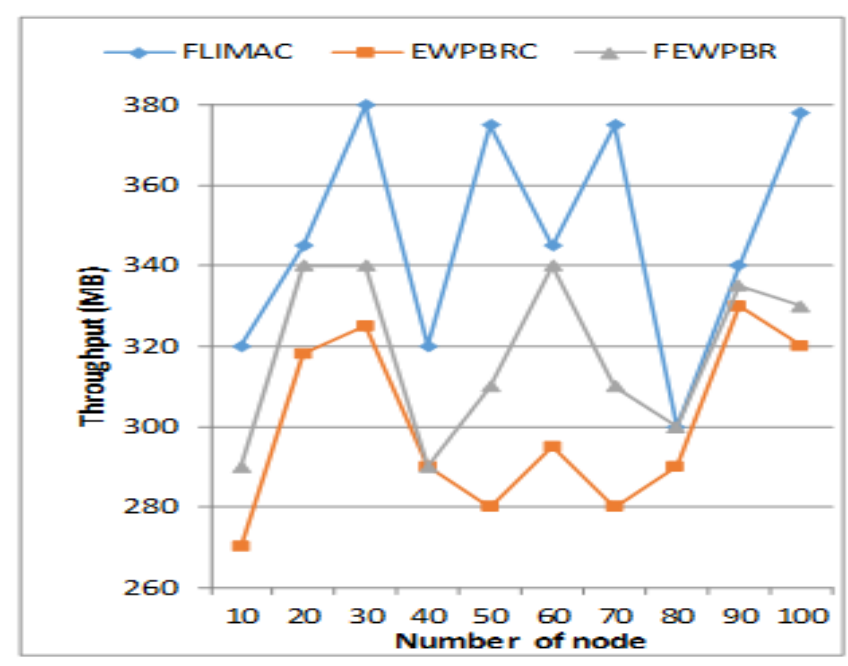

Table 5. Comparative analysis on Packet Loss Probability (\%)

\begin{tabular}{|l|l|l|l|}
\hline No.of Nodes & FLIMAC & EWPBRC & FEWPBR \\
\hline 10 & 42 & 48 & 54 \\
\hline 20 & 58 & 69 & 83 \\
\hline 30 & 55 & 62 & 65 \\
\hline 40 & 50 & 66 & 74 \\
\hline 50 & 55 & 57 & 68 \\
\hline 60 & 49 & 72 & 70 \\
\hline 70 & 56 & 54 & 60 \\
\hline 80 & 46 & 78 & 80 \\
\hline 90 & 53 & 55 & 62 \\
\hline 100 & 48 & 61 & 85 \\
\hline
\end{tabular}

Graph 3: Runs Vs. Packet Loss Probability

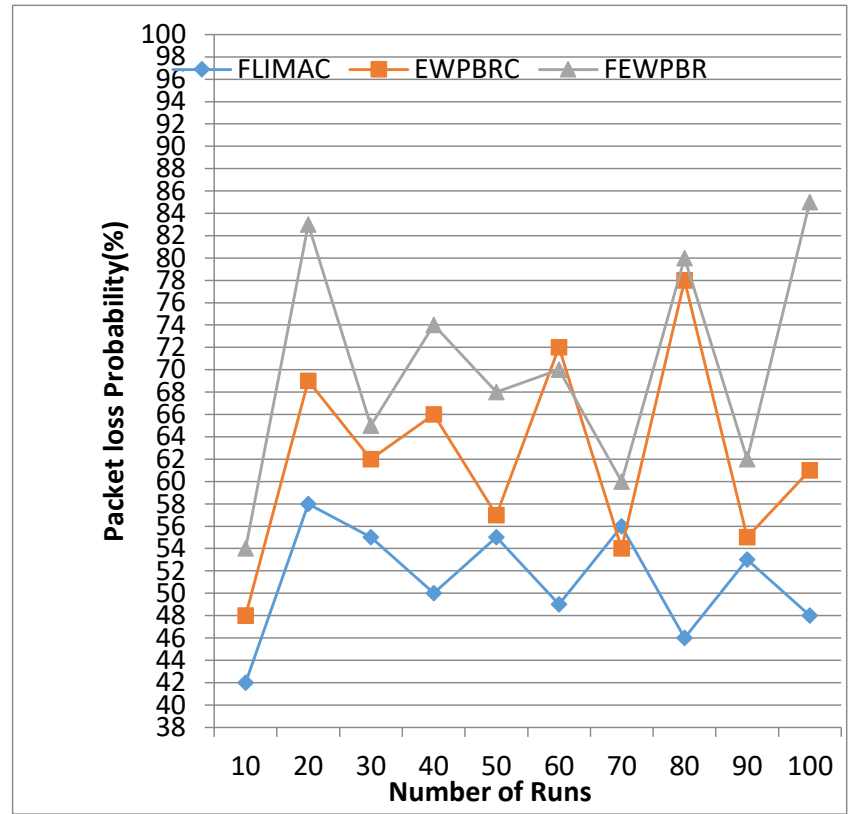

Table 3 shows the simulation of present work among various parameters with 20 runs and its average. The average Delay of the proposed work is $3.04 \%$ lower than FEWPBRC and similar to the EWPBRC. The Average throughput of the proposed work is $15.63 \%$ better than EWPBRC and $9.38 \%$ better than FEWPBRC. The typical packet loss of proposed work (FLIMAC) is $20.76 \%$ lower than FEWPBR and $11.3 \%$ lower than EWPBRC is $9.44 \%$. 
Table 6. Overall performance of the protocols with delay, throughput and packet loss

\begin{tabular}{|l|l|l|l|}
\hline $\begin{array}{l}\text { Performance } \\
\text { metrics }\end{array}$ & EWPBRC & FEWPBR & FLIMAC \\
\hline Delay & 0.329578 & 0.33456 & 0.32625 \\
\hline Throughput & 270.1036 & 290.1256 & 320.0144 \\
\hline Loss\% & 48.1009 & 53.0081 & 42.6153 \\
\hline
\end{tabular}

Table 4 describes the Comparative analysis on Throughput of FLIMAC has better performance when compared with EWPBRC and FEWPBR.

Table 5 clearly shows the Comparative analysis on Packet Loss Probability (\%) on various nodes. It is clearly shown in the graph 3 that the loss of packets using proposed protocol FLIMAC is less when compared with EWPBRC.

Table 6. Represents the total performance of the protocols with following metrics such as delay, throughput and loss where in delay of FLIMAC 0.32, throughput is 320 which remains high among the others and packet loss is low rather than the other protocols i.e 42.61 .

\section{Conclussion}

In this research, we propose a Fuzzy logic based Improved Medium access controller for Congestion Control and Prediction the Systems in WSNs. The MAC protocol controls information packet traffic congestion in the network. The proposed Improved MAC Algorithm links traffic rate measurement and dynamic queue management to attain energy competence and expand QoS Congestion Estimation and Data Rate Adjustment. Our proposed model compare with EWPBRC and FEWPBR protocols and show that the average Delay of the proposed work is 3.04\% lower than FEWPBRC and similar to the EWPBRC. The Average throughput of the proposed work is $15.63 \%$ better than EWPBRC and 9.38\% better than FEWPBRC. The typical packet loss of proposed work (FLIMAC) is $20.76 \%$ lower than FEWPBR and $11.3 \%$ lower than EWPBRC is $9.44 \%$. As part of the Future work, we suggest extending this work for large area network for avoiding congestion and improve the QoS..

\section{References}

[1] R. Ramesh, S. Kumara Ghuru, Cost measures of fuzzy batch arrival queuing model by ranking function method, Int. J. Sci. Res. 4 (2277-8179) (2015) 234-238.

[2] V. Ashok Kumar, A membership function solution approach to fuzzy queue with Erlang service model, Int. J. Math. Sci. Appl. 1 (2) (2011) 881-891.

[3] Zadeh, L. A. (1996). "Fuzzy logic = computing with words". IEEE Transactions on Fuzzy Systems. 4 (2): 103-111. doi:10.1109/91.493904.
[4] M. Chen, X. Zhu, H. Zhu, Service adaptively medium access control algorithm based on fuzzy logical for energy harvesting wireless sensor networks, Int. J. Netw. 9 (12) (2016).

[5] J. Patel, N. Khare, Information \& communications technology express, Indian J. Sci. Technol. 2 (3) (2012) 395-399.

[6] R. Rasmesh, S. Kumara Ghuru, Analysis of performance measures of fuzzy queuing model with an unreliable server using ranking function method, Int. J. Adv. Comput. Sci. Technol. 3 (10) (2014) 44-445.

[7] R. Srinivasan, Fuzzy queuing model using DSW algorithm, Int. J. Adv. Res. Math. Appl. 1 (1) (2014) 57-62.

[8] V. Jain, S. Agarwal, K. Goswami, Priority based fuzzy decision packet scheduling algorithm for QoS in wireless sensor network, Int. J. Comput. Appl. 97 (3) (2014) 28-31.

[9] J. Devaraj, D. Jaya lakshmi, A fuzzy approach to priority queues, Int. J. Fuzzy Math. Syst. 2 (4) (2012) 479-488.

[10] M. Hatamian, M. Almasi, M. Asadboland, M. Hatamian, H. Barati, Congestion-aware routing and fuzzy-based rate controller for wireless sensor networks, J. Appl. Wireless Commun. 25 (1) (2016).

[11] N. Kaur, D. Kumar, A review on priority based congestion control protocols for wireless sensor networks, Int. J. Adv. Res. Comput. Sci. Softw. Eng. 5 (6) (2015) 1420-1426.

[12] M. Hatamian, M. Almasi Bardmily, M. Asadboland, M. Hatamian, H. Barati, Congestionaware routing and fuzzy-based rate controller for wireless sensor networks, Radio Eng. 25 (1) (2016).

[13] J. Bhar, M. Zaidi, R. Ouni, S. Nasri, Performance evaluation of fuzzy controller for traffic stabilization, in: Int. Conf. on Signals, Circuits and Systems, 2008, pp. 1-5.

[14] S. Singh, S. Chand, B. Kuma, Energy efficient clustering protocol using fuzzy logic for heterogeneous WSNs, Int. J. Wireless Pers. Commun. (2015) Springer Science+BusinessMedia, New York.

[15] Mayandi, Muthuselvi \& Pillai, Kavitha. (2016). Probabilistic QOS Aware Congestion Control in Wireless Multimedia Sensor Networks. Circuits and Systems. 07. 2081-2094. 10.4236/cs.2016.79181. 